\title{
Molecular Assembly and Self-Assembly: Molecular Nanoscience for Future Technologies
}

\author{
Michael de Wild ${ }^{\mathrm{a}}$, Simon Berner ${ }^{\mathrm{a}}$, Hitoshi Suzuki ${ }^{\mathrm{a}, \mathrm{b}}$, Luca Ramoino ${ }^{\mathrm{a}}$, Alexis Baratoffa, \\ and Thomas A. Jung ${ }^{\mathrm{a}, \mathrm{c}^{\star}}$
}

\begin{abstract}
In this review the emerging science of single molecules is discussed from the perspective of nanoscale molecular functions and devices. New methods for the controlled assembly of well-defined molecular nanostructures are presented: self assembly and single molecular positioning. The observation and selective modification of conformation, electronics, and molecular mechanics of individual molecules and molecular assemblies by scanning probes are demonstrated. To complement this scientific review, some of the possible consequences and visions for future developments are discussed, as far as they derive from the presented systems. Here, the prospects of nanoscale science to stimulate technological evolution are exemplified.
\end{abstract}

Keywords: Conformational switch · Molecular adsorption · Molecular nanotechnology from the bottom-up - Molecular self-assembly

\section{Nanoscale Science}

With the emergence of the prefix 'NANO' in science and technology, many established fields were re-visited and interdisciplinarily integrated to achieve new scientific and technological goals. In this article, we first give a brief account of the evolution of molecular nanoscience from its roots in 'biological systems', 'supramolecular chemistry' [1][2] and 'physical selfassembly and growth'. Then, in the main part of this review article, we introduce two new principles of molecular self-assembly and layering: 1) conformational adaptive epitaxy which is a consequence of a molecule's internal 'conformal' flexibility and

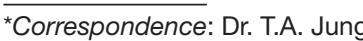

Paul Scherrer Institute

$\mathrm{CH}-5232$ Villigen PSI

Tel.: +41-56-310-4518

Fax: +41-56-310-2646

E-Mail: Thomas.Jung@psi.ch

a National Center of Competence in Research in

Nanoscience

Institute of Physics

University of Basel

Klingelbergstrasse 82

$\mathrm{CH}-4056$ Basel

${ }^{b}$ Kansai Adv. Res. Ctr.

Communications Research Laboratory

588-2 Iwaoka

Nishi-ku, Kobe 651-2492, Japan

cPaul Scherrer Institute

$\mathrm{CH}-5232$ Villigen
2) a new mode of self-assembly which leads to so-called 'self-intermixed monolayer phases' and derives from a delicate balance between electrostatic and Van der Waals interaction between static and fluctuating charge distributions of polar and polarizable molecules. In the final part of this article we discuss the presented scientific findings in terms of their technological potential and justify the high priority which has been given to molecular nano-technology by governmental funding agencies and corporate research strategies.

\section{Motivation}

Functional devices, as they rule present day electronics are mainly prepared by 'top-down' methods starting from the bulk silicon single crystal and involving only some 'bottom-up' elements such as material deposition and dopant implantation. While the cost-effective miniaturization of these devices remains the core target, hopes have evolved that 'bottom-up' assembly of functional molecular elements and devices will allow for smaller functional elements with the possibility of reproducible integration. Some of the milestones in this context are: the development of molecular electronics, the first imaging of atoms in real space by STM, the first artificial written structure 'IBM' by positioning of Xe atoms on an ultra-cold metal substrate. Functional molec- ular electronics such as organic light emitting diodes [3][4] and thin films transistors [5][6] have been manifested for 'thin films' many times thicker than one monolayer.

This article now tries to account for the research which has been targeted at the behavior of single functional molecules at contacting interfaces. In our view, this is a major challenge in today's 'molecular science' as it evolved from distinctively different traditional fields: bioscience, supramolecular chemistry and surface and interface science.

Molecular surface science was determined by the pioneering discoveries of Langmuir-Blodgett films [7-9], selfassembled monolayers [10-12] and the first studies of molecular epitaxy and layering in ultra-high vacuum [13]. Molecular layer self-assembly has been the basis for new applications like micro contact printing [14]. A universal definition of self-assembly in the context of this introduction was coined by G.M. Whitesides [15]:

'Molecular self-assembly is the spontaneous association of molecules under equilibrium conditions into stable, structurally well-defined aggregates joined by non-covalent bonds. Molecular selfassembly is ubiquitous in biological systems and underlies the formation of a wide variety of complex biological structures.' 


\section{Individual Molecules and Molecular Layers in Contact}

Single molecules or molecular assemblies with functional properties need to be supported and addressed by - presumably electronic - contacts. While molecules in liquid or gas remain unidentifiable within their statistic entity, their deposition by sublimation or evaporation on a well-prepared and atomically clean substrate (for an example see [16]) permits individual identification and tracking. This is a prerequisite for the assembly and operation of molecules into single molecular devices, whatever function we envision. Self-organized nanostructures of an even higher complexity can be assembled by using substrates which are themselves produced by heteroepitaxial self-organization: Periodic (vicinal) step edges provide a template for selective decoration of 'nanowire patterns'. Material systems with mixed magnetic/ nonmagnetic metal/insulator or semi-conducting properties have been realized [17] and chemically selective deposition of a molecular adsorbate has been demonstrated [18]. After immobilization of molecular sub-monolayers on such well-prepared and characterized substrates, scanning probe microscopy (SPM) provides the 'eyes' and the 'hands' for performing experiments with individual molecules and molecular assemblies. A large number of innovations, among them the above-mentioned thiol-derived SAM and the LB systems, were discovered and demonstrated without the assistance of SPM. Here, however, SPM provided a more detailed picture of the mechanisms on the atomic or molecular level and helped to gain control of many parameters like for example defect densities [19]. Beyond imaging, SPM also allows the selective application of electric fields, cur- rents and a variety of forces to manipulate atomic or molecular objects. This review focuses on the self-organization of a wellknown class of molecules, $\mathrm{C}_{60}$ Buckminster fullerenes (Fig. 1a), and porphyrins (Fig. 1b) as well as their close relatives, substituted phthalocyanines (Fig. 1c). All the layers shown were prepared by sublimation in ultra-high vacuum where the lack of a gas or solvent environment enables direct comparison of the experimental results with numerical simulations. 'Atomically clean' substrates were prepared by using surface science techniques: sputter-anneal cleaning cycles followed by characterization by LEED, XPS and STM.

\section{STM: The Eye to Investigate Molecular Epitaxy and Layering}

A prerequisite for the formation of epitaxial layers is the diffusive mobility of the adsorbate during or after the deposition of molecular ad-layers. On semiconductor substrates, often the strong and directed covalent bonds between the adsorbate and the adsorbent atoms inhibit the growth of periodic and defect-free layers. There, molecules have been imaged and show interesting characteristics which for example allow the molecular orientation and bonding to be identified [20-22]. In the case of metal substrates with lower reactivity like the noble metals $\mathrm{Au}, \mathrm{Ag}$ or $\mathrm{Cu}$, the diffusiveness of the molecule on the substrate allows the observation and analysis of quite different phenomena: The molecules 'feel' the corrugation of the substrate, and the lateral random-walk diffusion of a single adsorbate is determined by the corrugation of the potential energy surface (surface potential) of the adsorbate-substrate complex and by the kinetic energy available for hopping between stable adsorption sites. For Cu-TBPP adsorption on $\mathrm{Ag}(110)$ a complex adsorption behavior with fast diffusing precursor and slowly moving final adsorption states has been observed (Fig. 2 of [23]). The diffusion of the adsorbed molecule is confined to discontinuous one-dimensional motion within atomic trenches formed by an adsorbate induced $6 \times 1$ reconstruction of the substrate which is accompanied by conformational flexure of the molecule. The change of the diffusion pattern here is caused by the different surface potential. Alternatively, Schunack et. al. [24] have demonstrated that two similar organic molecules DC and HtBDC on the $\mathrm{Cu}(110)$ surface exhibit distinctively different diffusion behavior. This is a consequence of the altered substrate-adsorbate interaction by the additional substituent of the HtBDC which is in contact with the adsorbent. Depending on temperature and inter-molecular interaction, condensation of 2D islands with lattice registry, or more diffusive gas phases can be identified. Fig. 2a shows the condensation of a small molecular Cu-TBPP island in a chessboard-like overlayer structure. The opposite case is shown in Fig. 2b, where we can identify a 2D supramolecular island of SubPc which coexists with a 2D mobile gas phase. Notably, from the analysis of time-lapse imaging sequences, the hopping rate of the mole-cules was estimated to be in the order of $1 / 30 \mathrm{sec}^{-1}$ at room temperature. A detailed account of 2D mobile phases and of mole-cular diffusion on solid substrates is given in a recent paper by S. Berner et al. [26]. Molecular 'self-organization' generally leads to $2 \mathrm{D}$ super-structure islands with the lattice registry dictated by the substrate, and are quite different in spacing and symmetry from the bulk.
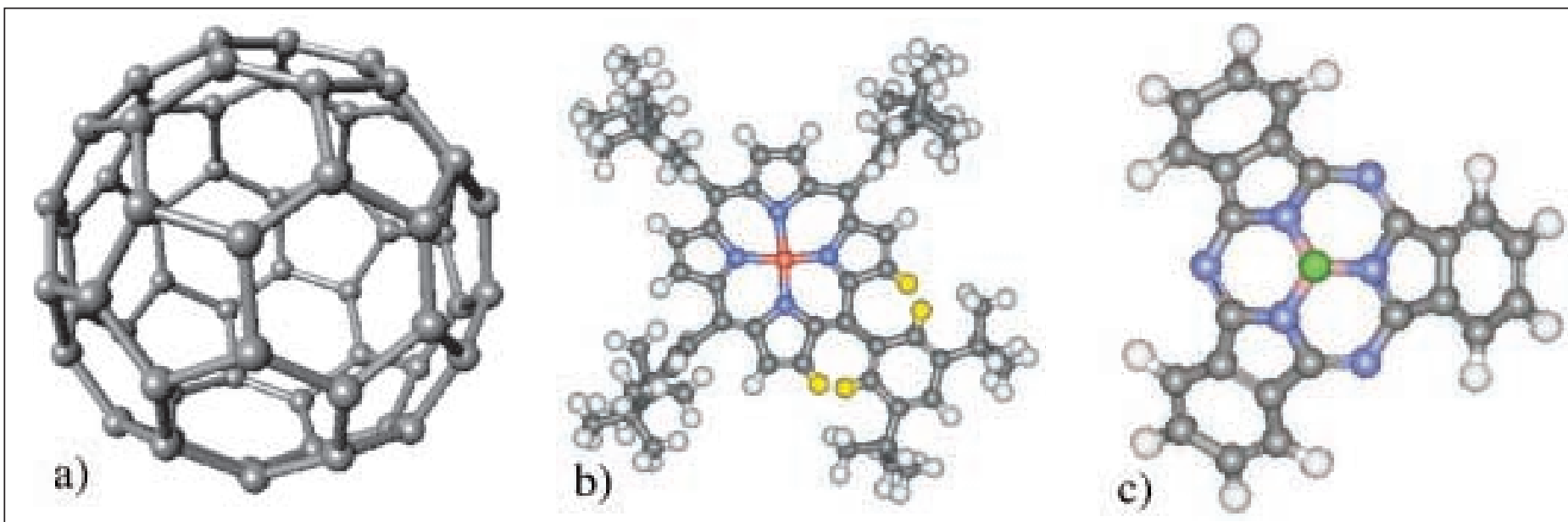

Fig. 1. Chemical structure of a) the Buckminster carbon fullerene $\mathrm{C}_{60}$, b) Cu-tetra-[3,5 di-t-butylphenyl]-porphyrin (Cu-TBPP) and c) chloro[subphthalocyaninato]boron(III) (SubPc). The molecular diameters are $1 \mathrm{~nm}, 2 \mathrm{~nm}$ and $1.5 \mathrm{~nm}$, respectively, and all three molecules have large delocalized $\pi$-systems which are responsible for the Van der Waals attraction between the adsorbates and the substrates. 


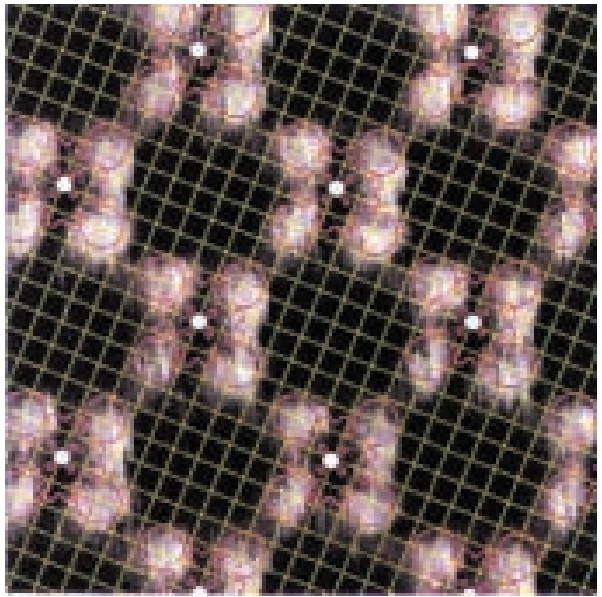

a)

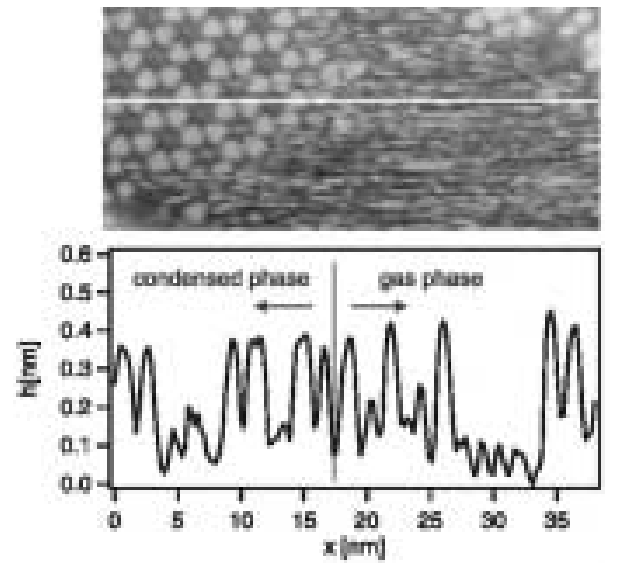

b)

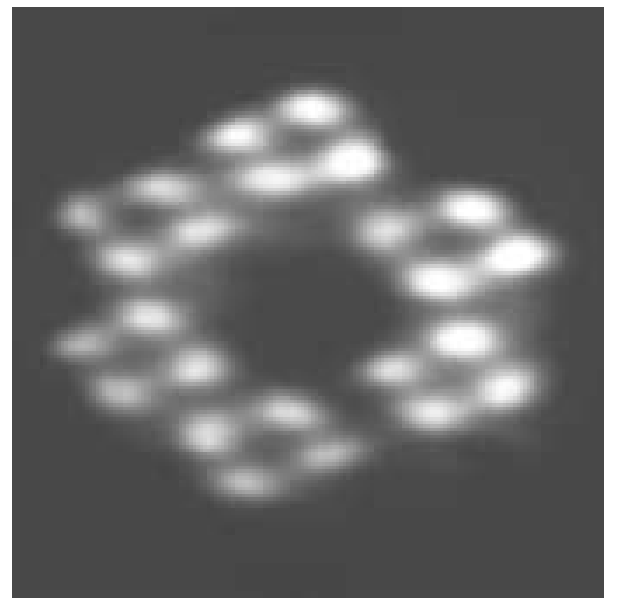

c)

Fig. 2. a) STM image of a condensed Cu-TBPP-island on $\mathrm{Cu}(100)$ after annealing at $250{ }^{\circ} \mathrm{C}$. Each of the molecules is resolved as four bright lobes with square symmetry. The positions of the molecular units in the two-dimensional overlayer is outlined [25]. Figure reprinted from Surface Science [25] with permission of Elsevier Science. b) Condensed SubPc-island on Ag(111) surrounded by a 2D gas-phase. The white line shows the location of the cross section (bottom part), which is parallel to the fast scanning direction: Single molecules are clearly visible, also in the noisy pattern of the gas-phase. This strongly suggests that the molecules can hop to adjacent adsorption sites, but that the residence time on a particular adsorption site is comparable to the time required by the scanning tip to pass over a single molecule. Scan range $38.4 \mathrm{~nm} \times 18.9 \mathrm{~nm}$, sample bias U $=0.85 \mathrm{~V}$, tunneling current I = $12 \mathrm{pA}$. Figure reprinted from Chem. Phys. Letts. [26] with permission of Elsevier Science. c) Image of an artificially created supramolecular structure, assembled by a STM tip. To move individual molecules, the tip is placed near one molecule, and the tunneling parameters are changed to 'manipulation'. Then the tip is moved to the desired direction across the position of the previously imaged molecule. After switching back to 'imaging' mode, the scan reveals a displacement of the molecule. The positions of all other molecules remain unaffected. After moving all molecules to the chosen places, the artificial hexameric ring is constructed. Figure reprinted from Science [27] with kind permission. Copyright 1996, Am. Assoc. Adv. Sciences.

\section{STM: The Hands to Manipulate Single Molecules}

Beyond imaging and tracking of the individual diffusive adsorbates in thermodynamic equilibrium, SPM also allows the addressing of molecular objects in non-equilibrium scenarios. In Fig. 2c we show the successful assembly of the first supramolecular assembly by STM tip-induced molecular re-positioning acheived at room temperature. Here, the lateral diffusion barrier has been overcome by lateral thrusts of the STM tip in a 'high proximity' mode at low gap voltages and high currents. By comparison of numerical simulations and experimental data, it has been shown that the conformational flexibility of the $\mathrm{Cu}$ TBPP molecules by torsion of the phenylporphyrin bond leads to a significant lowering of the diffusion barrier of this molecule on the $\mathrm{Cu}(100)$ substrate. The details of this technique can be read in [27].

Very much like the examples listed in the introduction, molecular re-positioning is dependent on a delicate balance of surface-molecular and intermolecular interaction forces. It is a prerequisite that the forces which are exerted by the tip in modification conditions can override the barrier for lateral displacement, while this barrier is high enough to resist room temperature diffusion. In addition to the repositioning of the flexible poprphyrins, also other sys- tems, mainly based on $\mathrm{C}_{60}$, have been found to meet these requirements [28][29]. Further details of the repositioning mechanism have been resolved by recording the tunneling current during the manipulation in experiments at low temperatures [30] and at room temperature.

Both epitaxy and repositioning, were first observed for noble-gas atoms at very low temperatures [31]. If adsorbed at all, these atoms and small molecules, such as $\mathrm{CO}$, tend to be too sticky to be played with at elevated temperatures. The larger molecules as they are presented here, which exhibit non-covalent binding, are better candidates to observe molecular diffusion, epitaxy and repositioning at room temperature. Another manipulation technique for single molecules are optical tweezers (see review 'Single Molecule Imaging and Manipulation' in this issue by A. Engel and M. Hegner [32]), where the objects are clamped with the aid of laser light.

\section{Conformational Self-Assembly and Dynamics: \\ Molecular Mechanics}

The special quality of a molecule is, however, that in its entity, it can be modified by synthetic chemists so that its interaction with both the tip and surface are specially designed and controlled, even at room temperature. By specifically designing the molecules, the force balance between the intermolecular aggregation and the many weak bonds to the substrate is maintained. Such 'synthetic' molecules can also be used as 'building blocks' for artificial supra-molecular structures as described in [33]. One main difference between molecules and atoms beside the afore-mentioned functionalization and stickiness is their more complex shape which essentially enables to excite internal 'conformational' degrees of freedom. Molecular conformation has been one of the keys in bio and life sciences, where analytical methods like XRD [34] and NMR [35] have enabled the visualization and understanding of complex proteins, their structure, function and dynamics. Together with other experimental techniques, these studies raised the issue of 'molecular conformation' and '3D molecular assembly' in the context of biomolecular aggregates and enzyme assemblies. For single molecular objects and small numbers of molecular assemblies, however, all these averaging diffraction and spectroscopy techniques fail due to lack of sensitivity. Some other techniques, like FIM, TEM, HREELS, etc. require a very selfselective sample preparation which allows only those experiments restricted to very special preparation conditions.

In the following paragraph, molecular conformation of surface adsorbates will be 
discussed, its recognition by scanning probe microscopy, as well as its adaptation, dynamics and switching. In Fig. 3, the conformational flexure and identification of $\mathrm{Cu}$-TBPP as it is deposited on a selection of metallic substrates is shown. For this molecule, the primary conformational degree of freedom consists of the rotation around the four phenyl-porphyrin dihedral angles. This rotation brings the $\pi$-systems of the ligands closer to overlap with the $\pi$-systems of the porphyrin-core. The full rotation is sterically hindered by the overlap of the hydrogen atoms of the porphyrin and the ligand (marked yellow in Fig. 1b). This conformational change can be observed when the ligands turn out and the porphyrin's $\pi$-system approaches the metallic substrates, so that the square symmetry of the molecule is broken. The aspect ratio of the rectangular shape of one molecule can be used to estimate the bond angle of each molecule [36].

Time lapse imaging sequences reveal that other molecular properties change in conjunction with conformational flexure and the molecules show conformational dynamics: The overlap of the $\pi$-electronic states with metallic states and the diffusibility of the molecules are the most prominent examples. Fig. 4a demonstrates that conformational flexure can go beyond

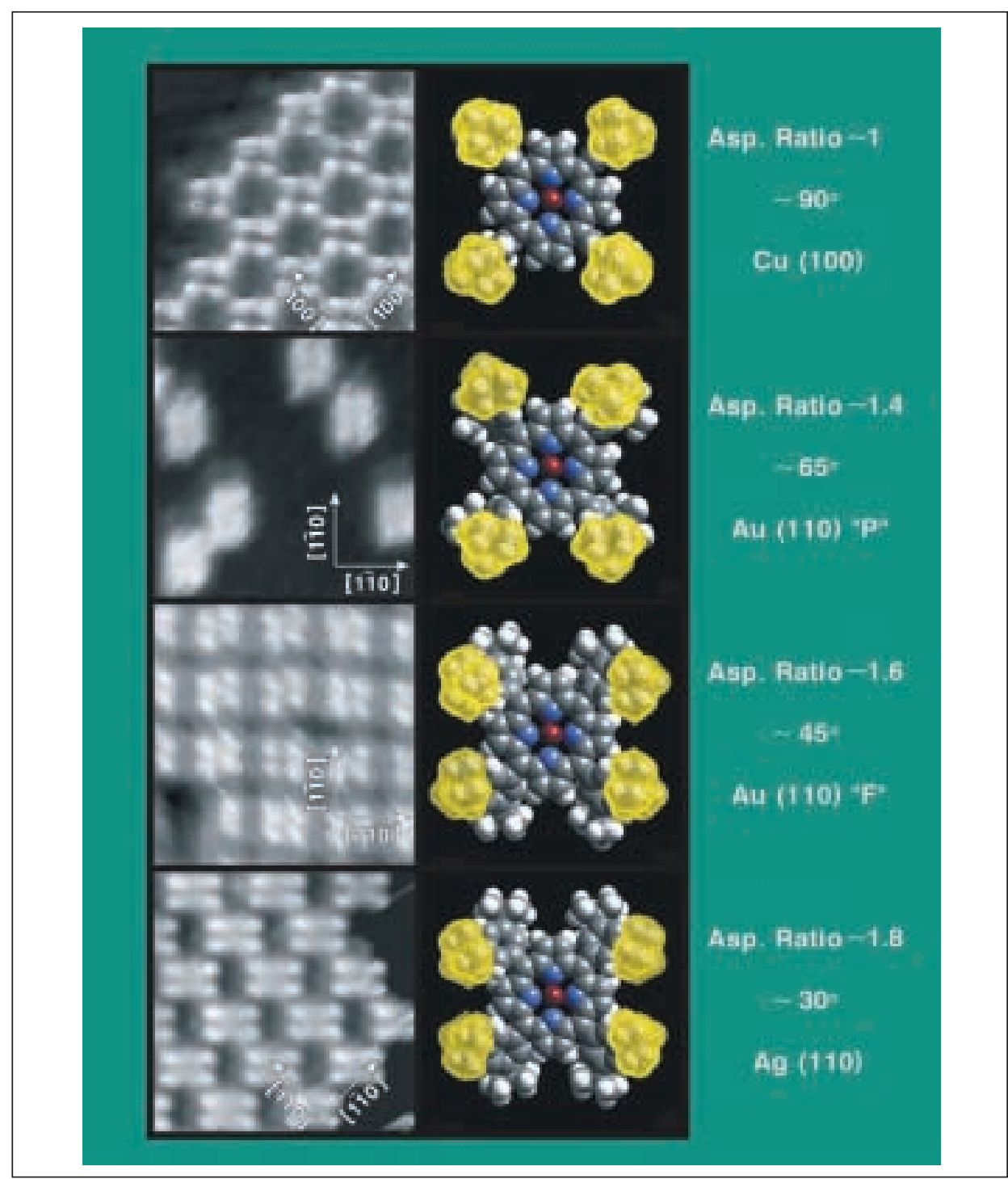

Fig. 3. Molecular 'epitaxial self-assembly' on a selection of metallic substrates. The overlayer symmetry and the orientation of each supramolecular ad-layer island can be identified from the high (sub-molecular) resolution STM data. The four-lobed quadrilateral forms with varying, substrate-dependent aspect ratio are clearly visible in the STM data (left-hand column). The lobes can be identified as the uppermost $t$-butyl groups of each of the four large $(\sim 1 \mathrm{~nm})$ substituents. In the models shown in the center column, the four bond angles of the phenyl-porphyrin bonds of each molecule have been determined such that the conformation of the model corresponds to STM data. The experimentally determined aspect ratio and the resulting bond angles are listed in the right-hand column, together with the substrate type and orientation. This contrast change reflects shifts of the porphyrin groups' electronic states and their coupling to the substrate. Figure adapted from Nature [36] with kind permission. Copyright (1997) Macmillan Publishers Ltd. a static phenomenon. The 'asymmetric' conformation of $\mathrm{Cu}$-TBPP within a monolayer as deposited on $\operatorname{Ag}(100)$ is changing repeatedly within a sequence of images. Here we speculate that the overall strain in the monolayer, which is a consequence of the strong molecule-substrate interaction, leads to an asymmetric conformation (Fig. 4b) because the substituents can more closely pack in this conformation. This view is supported by the observation that neighboring molecules show correlated shape changes: The 'high' substituent hardly exists in the nearest neighbor positions.

These results give clear evidence about the flexure and dynamics of a molecule's conformation. In our ongoing studies, we have also reproducibly observed the repeated pinning and de-pinning of $\mathrm{Cu}-\mathrm{TBPP}$ molecules by STM [37] and two other groups have further refined the current picture of the underlying process by performing these experiments at ultra-low temperatures [38] and by using the atomic force microscope (AFM) to measure the forces in the underlying processes [39]. With the AFM, the force to bend down a single leg by the pushing tip can directly be measured.

In a more general sense, the observation of conformational effects for single molecules is an astounding achievement in experimental molecular science which may give us even deeper insight into processes which occur at surfaces and play a fundamental role in catalysis, molecular electronics opto-electronics and tribology. For the nanoscale sciences most importantly, the relation between chemical 'structure' and property or 'function' has to be studied for technology-relevant ad-molecular layers and substrates. This is in close analogy to one of the paradigms of molecular biology, with one additional complication: molecular nanostructures for electronic, opto-electronic and mechanic operation need to be supported and contacted. The findings presented in this paper suggest that the property of a nanoscale molecular device is a joint property of the molecule and the contacting interface, as the conformational adaptation and shape of molecules are the product of the adsorbate's atomic structure and the contacting molecule.

\section{Self-intermixed Monolayer Phases}

While there is powerful means to reposition atoms and molecules at surfaces from the bottom up, it remains a challenge to handle a large number of atoms to organize them into structures with a high level of complexity. In spite of powerful develop- 


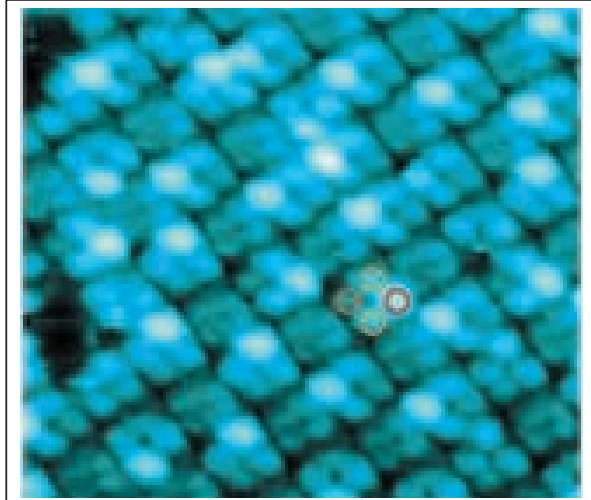

a)

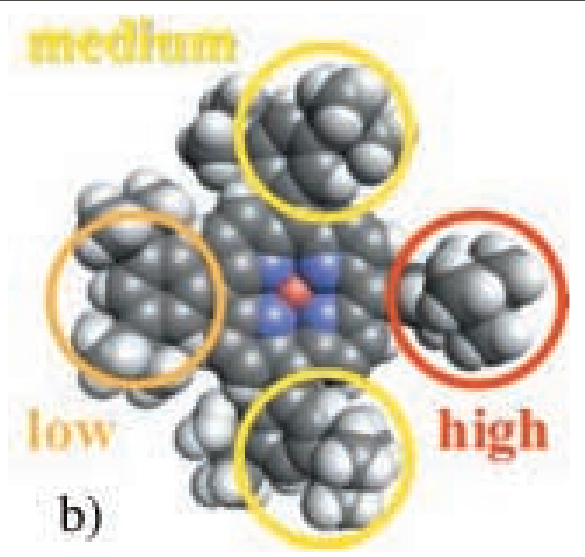

Fig. 4. a) STM image of a condensed Cu-TBPP-island on Ag(100). The molecules are adsorbed with asymmetric conformation. Individual ligands are turned with different angles and therefore appear with specific brightness in the STM images (the three observed heights are marked with circles). Image size: $12 \times 12 \mathrm{~nm}^{2}$. Sample bias $U=1 \mathrm{~V}$, tunneling current I $=40 \mathrm{pA}$. b) Model of the deformed molecule, adapted to the $\mathrm{Ag}(100)$ surface, shows the rotation of the TBP-groups around the $\sigma$-bonds.

ments in SPM parallelization like the IBM Millipede ${ }^{\odot}$ device, the piece-by-piece assembly and integration of functional nanostructures and devices is not a currently promising approach for the assembly of integrated molecular structures. In a technological sense the bottle-neck is the 'speed' or bandwidth $(\sim \mathrm{kHz})$ of our current tools, the scanning probe microscopes, which serve as the major instrumental links between the nano and the human world. Just to give one example:

To build a device with $10^{6}$ elements in a Gedankenexperiment (for example $\mathrm{Cu}$ TBPP porphyrins as switches) by positioning 'molecule-by-molecule', we would need 1 million 'strokes'. Each of these 'strokes' would require a molecular 'drag' and 'drop' procedure which at the moment can be achieved in just under one minute for each molecule, which leaves our Gedankenexperiment to be achieved on the timescale on the order of 700 days when using 'current sequential nanotechnology'. This is undoubtedly inadequate to construct a complex device with millions of elements (a state of the art Pentium 4 consists of 42 million transistors (MOSFETS) that work at $2.2 \mathrm{GHz}$ ). This is the flip side of the visionary statement 'There is plenty of room at the bottom' by R.P. Feynman in the 60's which we would translate to 'There are plenty of atoms in the wiring for submicrometer technology'. While we have reached the bottom in our current research, it remains difficult to make both ends meet and communicate from the level of single atoms and molecules up to the level of integrated electronic structures and macroscopic wires.

A promising way to overcome this obstacle is the self-assembly of mesoscopic structures. A completely new scheme for the self-organization of molecular patterns at the solid-vacuum interface has been recently discovered [40]: Unlike all other modes of molecular self-assembly, the molecules here form 2D periodic superstructures with an exceptionally high fraction of vacant adsorption sites. This is achieved by co-sublimation of two different molecules, one polar, the other highly polarizable, on a relatively inert metallic substrate in UHV. The two components, chloro-[subphthalocyaninato]boron(III) (SubPc) (see Fig. 1c) and carbon fullerene $\left(\mathrm{C}_{60}\right)$ (see Fig. 1a), co-deposited onto $\mathrm{Ag}$ (III) show a spectacular growth behavior. The growth and selforganization of this mixed monolayer is completely different from the pure monolayer islands of either component sublimed onto the same substrate [26]. In typical room-temperature STM images (left hand side of Fig. 5), individual molecules can be count see [40]. clearly identified by their size and characteristic triangular or spherical shapes which are in agreement with STM studies of the single-component monolayers. In the proposed model (right hand side of Fig. 5), the positions, orientations and shapes of the molecules can be seen. Due to attractive intermolecular interactions, the two kinds of molecules are periodically intermixed and not segregated into two separate phases: The $\mathrm{C}_{60}$ molecules (red) are arranged in quasi-linear rows, whereas the SubPc molecules (blue) form a zigzag spacer row. The self-intermixed assembly observed here relies crucially on sufficient mobility of each component at the deposition temperature. Remarkably, a significant fraction of the surface remains uncovered between the SubPc molecules, which leads to a loosely packed superstructure (the underlying $\mathrm{Ag}$ substrate appears black). The feasibility to manipulate molecules within these spacings opens the possibility to store data in specific molecular patterns at room temperature.

\section{Nanotechnology: Opportunities and Visions on Scientific Grounds}

To conclude, there are ample possibilities to organize and observe single, identifiable molecular objects when they are organized at surfaces. Using SPM as the 'eyes and hands', molecular organization and diffusion can be observed, molecules can be locally organized and probed in their electronic, mechanic and optic interaction with the environment. In particular, the observed time lapse imaging sequences provide visual information about diffusion and conformational dynamics which would not have been imagined before the invention of SPM, and only believed after many years of

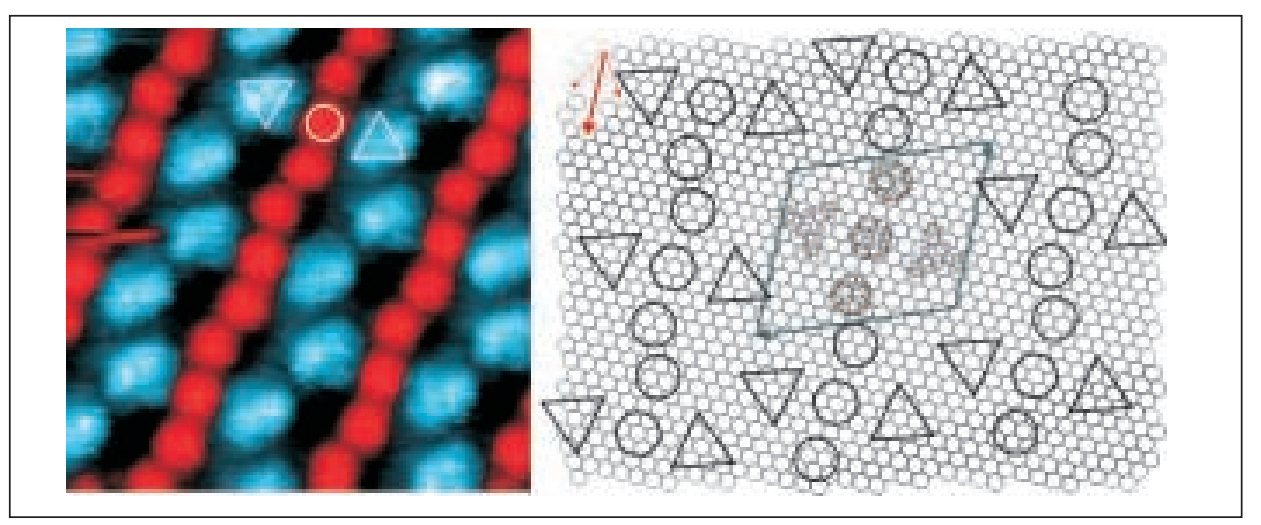

Fig. 5. Self-intermixed monolayer phase: $\mathrm{C}_{60}$ and SubPc (see Fig. 1a and c) codeposited on $\mathrm{Ag}(111)$, intermix and form a strongly anisotropic pattern. The $\mathrm{C}_{60} \mathrm{~s}$ are adsorbed in a quasi linear arrangement, and the SubPcs act as spacers between these $\mathrm{C}_{60}$-lines. Image size: $9.7 \times 9.7 \mathrm{~nm}^{2}$. Sample bias $U=1.9 \mathrm{~V}$, tunneling current $\mathrm{I}=20 \mathrm{pA}$. On the right hand side, the proposed model of the registry on $\mathrm{Ag}(111)$ is shown: The positions of the $\mathrm{C}_{60}$ and SubPc molecules and the orientations of the latter are depicted inside the unit cell. For a more detailed ac- 
experimental consolidation. The rapid evolution of the field of nanotechnology and the collaboration of scientists from many disciplines has triggered many hopes. To produce ultra-small structures which provide some functionality to our decimeter world, two key problems need to be addressed: First ultra-small devices need to be manufactured, and second, they need to be interconnected and 'wired' at least to the micrometer level which is accessible by 'state-of-the-art' technology.

\section{Molecular Level Data Storage}

In a visionary context and on the basis of the above described 'key' inventions, it seems plausible that there may be technological implementations of single molecule storage on materials as suggested by the above described 'SIMP' material. The assignment of $1 \mathrm{~nm}$-sized bits and bytes to the zigzag rows is straightforward, while the $\mathrm{read} / \mathrm{write}$ rates are further away from competitive technology.

\section{Molecular Opto-Electronics}

For fundamental and applied reasons, molecular bi-stability e.g. conformational and positional switching as shown in this review, is of high scientific and technological interest. The reason here is that on a molecular level, molecular mechanics and electronics share the same origin. The electrons which form the electronic bonds, determine ionization potentials and atomic (mechanical) bonding, and therefore optic and electronic properties of the molecular bond are altered. Here, it is extremely important to point out that this individual bond is generally interacting with other bonds inside the molecule and of course with the substrate. This is one of the most important results of the discussed research: The positional and conformational bi-stability of all the above systems is not a consequence of a synthesized 'molecular switch', but of a complex behavior of a molecule when adsorbed in the surface potential of the substrate. This also connects to other surface effects like drastic changes in chemical reactivity (catalysis) and enantioselectivity. Future experimental and theoretical efforts towards nanoscale devices will have to investigate these complex interactions in more detail, in order to be able to predict functions for new, 'to be synthesized' systems.

To conclude, this article has given an account of our current 'scientific' knowledge about a single molecule's 'behavior' when it is adsorbed on a solid substrate. Mechanic, electronic and optic properties are convoluted, since the origin is the chemical and physical bonding of the atoms within the molecule and the substrate Methodologically, it seems worthwhile to note that SPM-based techniques are the ONLY techniques which allow us to observe the conformation, electronics and mechanics of single molecules. To complement this scientific account, we have discussed some of the potential applications of "nanotechnology' as far as they derive from these examples. Most importantly, there is a huge potential for research and discovery, which has motivated National and International Research Programs to collaborate with Multi National corporations in one or the other field. The close collaboration of scientists and technologists from many different fields propels nanoscience and nano-technology.

\section{Acknowledgements}

Thomas Jung takes this opportunity to thank his tutors in physics chemistry: H.R. Christen, K. Wuethrich, R. Ernst, H.C. Siegmann, H.-J Guentherodt, P. Avouris, F. Himpsel, J. K. Gimzewsk, and H. Rohrer. We thank S. Schintke, R. Bennewitz, R. Schlittler, E. Meyer, and $H$. Yanagi for their support and collaboration. It is a pleasure to acknowledge A. Fischer for the careful proofreading of the manuscript and C. Bolliger for advice on copyright issues.

We thank T. Kamikado, S. Yokoyama, and S. Mashiko from the Kansai Advanced Research Center, Japan, for providing the porphyrin molecules and D. Schlettwein, W. Michaelis, H. Kliesch, and D. Wöhrle, Institut für Organische und Makromolekulare Chemie, Univ. Bremen and Oldenburg, Germany, for the SubPc molecules.

This work was financially supported by the University of Basel, the Paul Scherrer Institut, the Swiss National Science Foundation, the TopNano 21-Project, and the NCCR on 'Nanoscale Science'

Received: September 3, 2002

[1] J.-M. Lehn, Science 1993, 260, 1762.

[2] J.-M. Lehn, VCH Weinheim, 1995, vol. 277, ISBN 3-527-29312-4.

[3] J.H. Burroughes, D.D.C. Bradley, A.R. Brown, R.N. Marks, K. Mackay, R.H. Friend, P.L. Burns, A.B. Holmes, Nature 1990, 347, 539.

[4] S.R. Forrest, Chem. Rev. 1997, 97, 1793.

[5] H.E. Katz, J. Mat. Chem. 1997, 7, 369.

[6] G. Horowitz, Adv. Mat. 1998, 10, 365.

[7] G.G. Roberts, Langmuir-Blodgett Films, Plenum Press, New York, 1990.

[8] A. Ulman, 'Ultrathin organic films', from 'Langmuir-Blodgett to Self-Assembly', Academic Press, Inc., San Diego, 1991.

[9] D.Y. Takamoto, E. Aydil, J.A. Zasadzinski, A.T. Ivanova, D.K. Schwartz, T.L. Yang, P.S. Cremer, Science 2001, 293, 1292.

[10] A. Ulman, Chem. Rev. 1996, 96, 1533.

[11] T. Takami, E. Delamarche, B. Michel, Langmuir 1995, 11, 3876.

[12] E. Meyer, R. Overney, R. Luethi, D. Brodbeck, L. Howald, J. Frommer, H.-J.Güntherodt, O. Wolter, M. Fujihira, H. Takano, Y. Gotoh, Thin Solid Films 1992, 220, 132.
[13] H. Ohtani, R.J. Wilson, S. Chiang, C.M. Mate, Phys. Rev. Lett. 1988, 60, 2398.

[14] R.J. Jackman, J.L. Wilbur, G.M. Whitesides, Science 1995, 269, 664.

[15] G.M. Whitesides, J.P. Mathias, C.T. Seto, Science 1991, 254, 1312.

[16] J.V. Barth, H. Brune, G. Ertl, R.J. Behm, Phys. Rev. B 1990, 42, 9307.

[17] F.J. Himpsel, T.A. Jung, A. Kirakosian, J.-L. Lin, D.Y. Petrovykh, H. Rauscher, J. Viernow, MRS Bulletin 1999, 24, 20.

[18] H. Rauscher, T.A. Jung, J.-L. Lin, A. Kirakosian, F.J. Himpsel, U. Rohr, K. Müllen, Chem. Phys. Lett. 1999, 303, 363.

[19] C. Schönenberger, A.J.M. SondagHuethorst, J. Jorritsma, L.G.J. Fokkink, Langmuir 1994, 10, 611.

[20] Y.W. Mo, Science 1993, 261, 886.

[21] R.J. Hamers, J.S. Hovis, S.K. Coulter, M.D. Ellison, D.F. Padowitz, Japanese Journal of Applied Physics 2000, 39, 4366.

[22] H. Yanagi, D. Schlettwein, H. Nakayama, T. Nishino, Phys. Rev. B 2000, 61, 1959.

[23] J.K. Gimzewski, T.A. Jung, M.T. Cuberes, R.R. Schlittler, Surface Science 1997, 386, 101.

[24] M. Schunack, T.R. Linderoth, F. Rosei, E. Laegsgaard, I. Stensgaard, F. Besenbacher, Phys. Rev. Lett. 2002, 88, 156102/1-4.

[25] J.K. Gimzewski, T.A. Jung, M.T. Cuberes, R.R. Schlittler, Surface Science 1997, 386, 101.

[26] S. Berner, M. Brunner, L. Ramoino, H. Suzuki, H.-J. Güntherodt, T.A. Jung, Chem. Phys. Lett. 2001, 348, 175.

[27] T.A. Jung, R.R. Schlittler, J.K. Gimzewski, H. Tang, C. Joachim, Science 1996, $271,181$.

[28] P.H. Beton, A. Dunn, P. Moriarty, Appl. Phys. Lett. 1995, 67, 1075.

[29] M.T. Cuberes, R.R. Schlittler, J.K. Gimzewski, Surface Science 1996, 371, 181.

[30] F. Moresco, G. Meyer, K.-H. Rieder, H. Tang, A. Goudron, C. Joachim, Phys. Rev. Lett. 2001, 87, 88302-1.

[31] D.M. Eigler, E.K. Schweizer, Nature 1990, 344, 524.

[32] M. Hegner, A. Engel, Chimia 2002, 56, 505.

[33] T. Yokoyama, S. Yokoyama, T. Kamikado, Shinro Mashiko, J. of Chem. Phys. 2001, $115,3814$.

[34] M.F. Perutz, H.C. Watson, H. Muirhead, R. Diamond, W. Bolton, Nature 1964, 203, 687.

[35] K. Wüthrich, Nature Struct. Biol. 2001, 8 , 923-925.

[36] T.A. Jung, R.R. Schlittler, J.K. Gimzewski, Nature 1997, 386, 696.

[37] R. R. Schlittler, T. A. Jung, J. K. Gimzewski, unpublished.

[38] F. Moresco, G. Meyer, K.-H. Rieder, H. Tang, A. Goudron, C. Joachim, Phys. Rev. Lett. 2001, 86, 672.

[39] C. Loppacher, M. Bammerlin, M. Guggisberg, E. Meyer, H.-J. Güntherodt, R. Schlittler, J.K. Gimzewski, Appl. Phys. A 2001, 72, 105 .

[40] M. de Wild, S. Berner, H. Suzuki, H. Yanagi, D. Schlettwein, S. Ivan, A. Baratoff, H.-J. Güntherodt, T.A. Jung, ChemPhys Chem 2002, accepted. 